\title{
The impact of coupled conductors in a dielectric material above an ideally conducting plane on the difference of board delay per unit length of even and odd modes
}

\author{
Pavel Yevgenyevich Orlov*, Talgat Rashitovich Gazizov, Aleksander Mikhailovich Zabolotsky
}

Tomsk State University of Control Systems and Radioelectronics, Tomsk, Russia

\section{ARTICLE INFO}

\section{Article history:}

Received 31 October 2016

Received in revised form

25 January 2017

Accepted 1 February 2017

\section{Keywords:}

Dielectric material

Board delays per unit length

Even and odd modes

Strip structures

\begin{abstract}
A B S T R A C T
Dielectric fill features may have a significant effect on the characteristics of both the cable and microstrip transmission lines. It is necessary to provide proper characteristic impedance of microstrip transmission lines to create a multi-layer printed circuit boards with digital, analog and RF circuits. The authors simulate the impact of the planar location of two conductors in a dielectric material above an ideally conducting plane on the board delays per unit length of transmission-line modes. The simulation showed the dependency of even and odd modes and their differences on the distance between the conductors during the change in the depth of immersion of conductors into the dielectric material. The behavior of obtained dependencies was explained. It was discovered that the difference of board delays changes sign and reaches high values (from - 0.8 to $1.5 \mathrm{~ns} / \mathrm{m}$ ) with close coupling. Obtained results can be used for further research in the field of electronics. In particular, for model filtering development based on microstrip lines. This will reduce the level of the ultrashort pulses problem.
\end{abstract}

(C) 2017 The Authors. Published by IASE. This is an open access article under the CC BY-NC-ND license (http://creativecommons.org/licenses/by-nc-nd/4.0/).

\section{Introduction}

Increased attention to cables and cable medium voltage lines can be explained by the fact that they constitute the majority of power cables in industrial enterprises and cities. That is why blackout will cause industrial sector's termination. Cable can be replaced on modern one with cured ethylene polymer to solve the problem as they have reached the end of their service life (Pavel et al., 2016).

However, replacement of entire cabling system will require several years and huge financial costs. Therefore, to extend installed cables' operation and maintenance, it is necessary to analyze characteristics of the dependencies of board delays per unit length of even and odd modes and their difference on the location of two conductors in a single dielectric above an ideally conducting plane.

Dielectric filling of transmission lines can affect their characteristics significantly. This is especially evident during high-frequency signals and a close coupling between line conductors. Therefore, coupled strip lines in a heterogeneous dielectric

\footnotetext{
* Corresponding Author.

Email Address: praetorian281@gmail.com (P. Y. Orlov) https://doi.org/10.21833/ijaas.2017.02.012

2313-626X/C 2017 The Authors. Published by IASE.

This is an open access article under the CC BY-NC-ND license

(http://creativecommons.org/licenses/by-nc-nd/4.0/)
}

filling are actively studied. After ordinary microstrips, studies concerned lines on a dielectric substrate, diversely located in air with respect to shields of various types. For example, the dependency of the odd and even modes and coupling coefficients on the distance between conductors was studied for strips enclosed by a rectangular shield (Allen and Este, 1972). The use of dielectric materials' heat treatment processes is aimed at energy use of electromagnetic super-high-frequency field as a heat source (Allen and Este, 1972). Conditions and charts were obtained for the coupling coefficients and wave impedance for suspended coupled lines, with which the velocities of even and odd modes coincide (Krasonperkin et al., 1983a); the peculiarities of impulse propagation in such lines were demonstrated (Krasonperkin et al., 1983b). Suspended and inverted lines were studied for dual dielectrics (Gazizov and Leontiev, 1997) via the sign of difference of capacitive and inductive coupling coefficients. Other studies concerned the mechanism of pulse signal distortion due to the discrepancy between the velocities of even and odd modes in a multilayer dielectric structure, during the change in distance between conductors (Gilb and Balanis, 1989). Important peculiarities of such distortions were discovered (Zabolotsky et al., 2006). However, certain dependencies of simple but widely used in practice conductors and dielectrics 
are understudied. For example, coupled lines, immersed into a single dielectric. The peculiarities of their interaction were partially considered in papers (Senthinathan et al., 1987; Muthana and Kroger, 2007). However, an additional study can help discover new regularities in the behavior of these lines' dependencies, in particular, useful modal phenomena, similar to the ones suggested for printed (Gazizov et al., 2012a;b) and cable (Gazizov et al., 2009; Gazizov and Zabolotsky, 2012; Orlov et al., 2013) structures.

The purpose of this paper is to study the dependencies of board delays per unit length of even and odd modes and their difference on the location of two conductors in a single dielectric above an ideally conducting plane, in particular, with a close coupling.

\section{Methods}

Board delays of even $\left(\tau_{e}\right)$ and odd $\left(\tau_{o}\right)$ modes, and their difference $\left(\tau_{e}-\tau_{o}\right)$ were calculated in the TALGAT system by the method of moments (Gazizov, 2001). The studied structure (Fig. 1) had unchanged parameters, usual for printed boards: 2 identical conductors (width $w=0.5 \mathrm{~mm}$, thickness $t=35 \mu \mathrm{m}$ ), planar-located in a single layer of dielectric (thickness $H=1 \mathrm{~mm}$, relative permittivity $\varepsilon_{r}=5.4$ ) above an ideally conducting plane (Simulation parameters: considered distance between the conductor and the edge of the structure $d=4 \mathrm{w}$, segment length $5 \mu \mathrm{m}$ ).

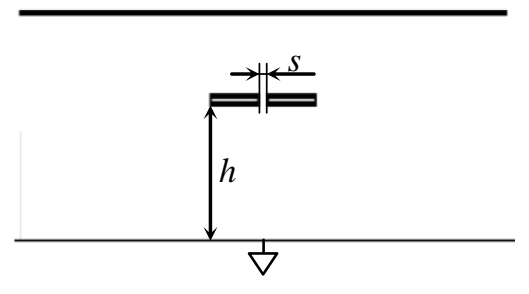

a

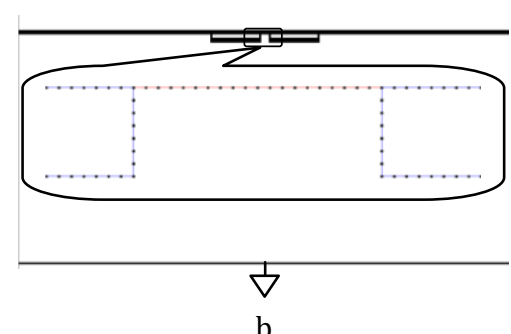

$\mathrm{b}$

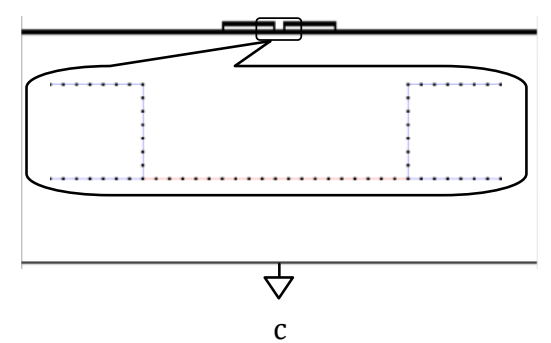

$c$

Fig. 1: Cross-sections of studied structures: conductors in a dielectric layer (a), conductors under (b) and above (c) the dielectric boundary with air (clearance boundaries are shown in magnified fragments)

The simulation allowed obtaining the dependencies on the distance between conductor edges $s$ with various distances of conductors from the ground plane $h$. Special cases of conductor location above and under the boundary of the dielectric with air required the simulation of individual structures (Fig. 1b for $h=0.965 \mathrm{~mm}$ and Fig. 1c for $h=1 \mathrm{~mm}$ ).

\section{Data, analysis, and results}

The dependencies $\tau_{e}, \tau_{o}$ and $\tau_{e}-\tau_{o}$, calculated for $h=0.2 ; 0.6 ; 0.9 ; 0.965 ; 1 \mathrm{~mm}$ within the range $s=0.1-5$ $\mathrm{mm}$ (up to loose coupling), are presented in Fig. 2a and $2 \mathrm{~b}$, while in the range $s=0.01-0.1 \mathrm{~mm}$ (with close coupling) in Fig. 2c and 2 d respectively.

Before presenting the detained analysis of dependencies, it should be noted that their nature generally depends on the electric-field distribution of the even and odd modes in the line cross-section. Indeed, with an even mode, an identical voltage is applied to both conductors of the studied structures with respect to the shield, while with an odd mode the voltage of opposite polarity is applied to one of the conductors.

The electric field is concentrated between the structure conductors, but the concentration of its lines of force in areas between two signal tracks and in areas between them and the shield varies significantly, depending on the mode and correlation of line parameters, including on the location of boundary lines of dielectrics. This leads to the greater concentration of this or that mode's field in the dielectric than in the air, therefore, the board delay per unit length of this mode will be greater. In particular, with the reduction of the clearance between signal tracks of the studied structures, the even mode field is increasingly forced out of the clearance, while the odd mode field becomes more concentrated in the clearance. At that, the proximity of the dielectric boundary clearance and air affects the reduction of the board delay per unit length. It should be noted that for the use of modal phenomena in practice, especially important are the sign, zero and maximum absolute values of board delay per unit length differences with appropriate regions of line parameters.

Now it is worth examining the calculated dependencies in detail. For clarity, it is expedient to start with the most commonly known case for $h=1$ $\mathrm{mm}$ (microstrip). From Fig. 2a one can see that the board delay per unit length of even mode $\left(\tau_{e}\right)$ is always greater than that of the odd mode $\left(\tau_{o}\right)$. This is caused by the fact that the even mode transits greater in the dielectric (between signal tracks and shields), than the odd mode (between signal tracks). The reduction of $s$ causes a slight increase of $\tau_{e}$, while $\tau_{o}$ reduces; moreover, the smaller $s$, the greater $\tau_{o}$ (Fig. 2c), which is caused by an increasingly greater concentration of the odd-mode electric field in the air clearance between the edges of signal tracks. It should be noted that this reduction of $\tau_{o}$, caused by the air in the clearance, leads to a rapid growth of $\tau_{e^{-}}$ $\tau_{o}$ with small $s$ : up to $0.7 \mathrm{~ns} / \mathrm{m}$ in Fig. $2 \mathrm{~b}$ or even up to $1.5 \mathrm{~ns} / \mathrm{m}$ in Fig. $2 \mathrm{~d}$. The indicative transit is that from $h=1 \mathrm{~mm}$ (Fig. 1c) to $h=0.965 \mathrm{~mm}$ (Fig. 1b), when the signal tracks are immersed in the dielectric at their depth of $35 \mu \mathrm{m}$. 

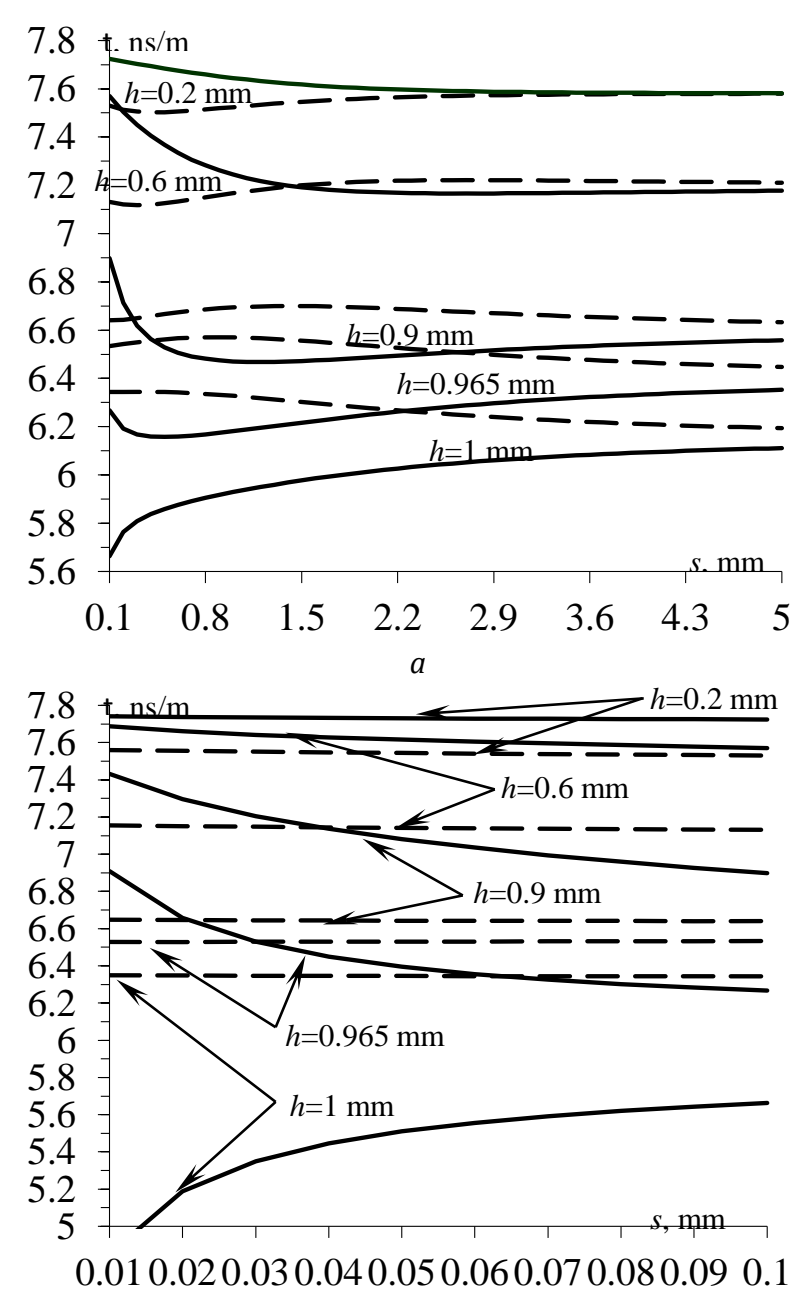

C
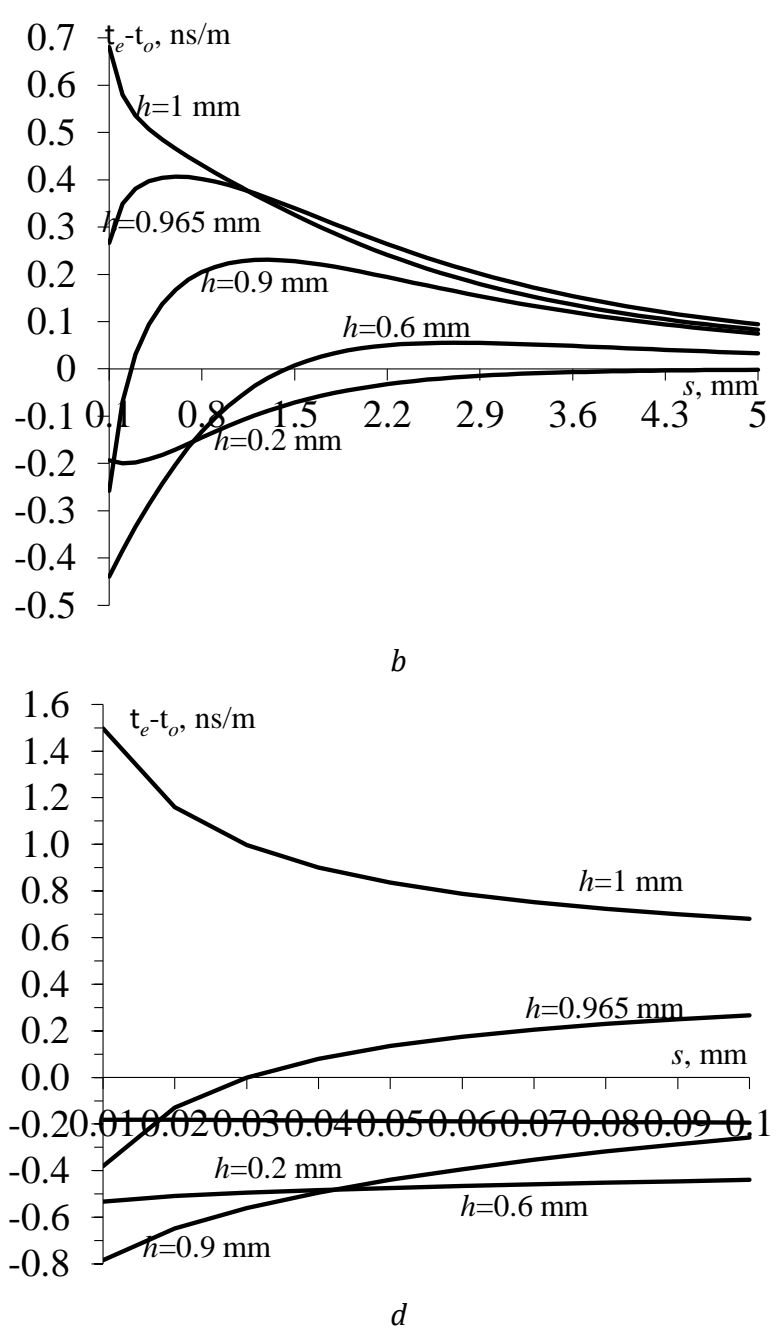

Fig. 2: Dependencies on s: $a, b-$ for $\tau e(--)$ and $\tau o(--) ; c, d-$ for $\tau e-\tau o$

From Fig. 2a one can see that $\tau_{e}$ and $\tau_{o}$ increase, which is natural, since both modes transit greater in the dielectric. However, it should be noted that in the $\tau_{o}$ dependency, an ascendancy area appeared with a decrease in $s$. It is possible to assume that it is caused by the filling of the gap with the dielectric. Now, extremum points appeared in the dependencies: a weakly pronounced maximum $\tau_{e} ;$ a stronger minimum $\tau_{o}$; a strongly pronounced maximum $\tau_{e}-\tau_{o}$. As a result, with a reduction of $s$, a point of intersection of dependencies $\tau_{e}, \tau_{o}$ (Fig. 2b) appears, the $\tau_{e}-\tau_{o}$ value decreases (Fig. 2b), assuming a zero or even negative values (Fig. $2 \mathrm{~d}$ ). The intersection of dependencies for $h=1 \mathrm{~mm}$ and $h=0.965 \mathrm{~mm}$ (Fig. 2b), which has its consequences, is also noteworthy. Further immersion of signal tracks into the dielectric (Fig. 1a) shifts the point of intersection of dependencies $\tau_{e}, \tau_{o}$ in the direction of greater $s$. This leads to significantly great (up to $-0.8 \mathrm{~ns} / \mathrm{m}$ ) negative values of $\tau_{e}-\tau_{o}$. Their dependency on $h$ is nonlinear, and is of interest for further studies.

\section{Discussion}

The known facts of positive $\tau_{e}-\tau_{o}$ value of coupled microstrip lines, its nullification by solid filling with the same dielectric, and negative value by coating with a dielectric with a greater $\varepsilon_{r}$ value somewhat hinder the possibility of obtaining a zero and negative $\tau_{e}-\tau_{o}$ value by immersing line conductors into the same dielectric with a reduction of $s$. It is physically explained by increasingly more pronounced, but opposite phenomena: the forcing out of the even-mode field from the clearance to the boundary with air and the concentration of the oddmode field in the clearance. Therefore, in the region of small $s$, the even mode is always slower than the odd mode, even with the deepest immersion, when both modes are increasingly decelerated.

It is necessary to provide predetermined characteristic impedance of microstrip transmission line to create an electronic circuit. Logic ground use as the next layer requires a very narrow band, which increases its relative width spread, as well as the spread of characteristic impedance. According to plane capacitor properties, increasing distance from the strip to the ground allows to increase the width of the strip (Gazizov and Zabolotsky, 2012).

Heat and mass exchange processes under nominal operating conditions determine power cables' transmission capacity. It is advisable to use the most well-known calculation model to determine power cables' overload capacity, namely exponential model of cable heating (Kosowsky, 2012). 
Modern electronics based on microelectronic components have an increased susceptibility to EMI, especially to ultrashort pulses, which are able to disturb the apparatus. We considered modal filtering technology to protect equipment from strong ultrashort pulse. It is based on the use of modal pulse signal decomposition in multiconductor transmission lines due to differences in modes' delays. Model filtering implementation is available on different structural levels of equipment, including by means of microstrip lines. That increases the protection from ultrashort pulses.

\section{Conclusion}

The article has revealed the dependence of even and odd modes and their differences on the distance between the conductors during the change in the depth of immersion of conductors into the dielectric material. It was found that the difference of board delays changes its operator and reaches high values - from 0.8 to $1.5 \mathrm{~ns} / \mathrm{m}$ with a close relationship.

This study was conducted for practically applied line parameter values, but the subset value was unchanged. This means that with their different values, the behavior of studied dependencies may change. However, it is possible to control these changes with a view to obtaining required characteristics. It is also useful to construct dependencies on other parameters.

Thus, this research describes characteristics of the dependencies of board delays per unit length of even and odd modes and their difference on the location of two conductors in a single dielectric above an ideally conducting plane and can be used for further research in the field of electronics.

\section{Acknowledgement}

The study was conducted with the financial help of the Ministry of Education and Science of the Russian Federation, under the basic part of governmental order No.2014/225 for project 769 and the Russian Foundation for Basic Research, under scientific project No.13-07-98017 r_siberia_a.

\section{References}

Allen JL and Este MF (1972). Broadside - coupled strips in a layered dielectric medium. IEEE Transactions on Microwave Theory and Techniques, 20(10): 662-669.

Gazizov TR (2001). Analytic expressions for MOM calculation of Capacitance matrix of two dimensional system of conductors and dielectrics having arbitrarily oriented boundaries. In the
IEEE International Symposium on Electromagnetic Compatibility (EMC), IEEE, Montreal, Canada, 1: 151-155. https://doi.org/10.1109/ISEMC.2001.950576

Gazizov TR and Leontiev NA (1997). Reduction of high-speed signal distortions in double-layered dielectric PCB interconnects. In the IEEE $6^{\text {th }}$ Topical Meeting on Electrical Performance of Electronic Packaging, IEEE: 67-69. https://doi.org/10.1109/EPEP.1997.634040

Gazizov TR and Zabolotsky AM (2012). Experimental results on UWB pulse propagation in low-voltage power cables with different cross sections. IEEE Transactions on Electromagnetic Compatibility, 54(1): 229-231.

Gazizov TR, Dolganov ES, and Zabolotsky AM (2012a). Modal filter as a device for electrostatic discharge protection of onboard computers and control units of space vehicles. Russian Physics Journal, 55(3): 282-286.

Gazizov TR, Zabolotsky AM, and Samotin IP (2009). Experimental results on ultra wide band pulse propagation in threeconductor power cables of flat and circular cross sections. In the International Siberian Conference on Control and Communications (SIBCON '09), IEEE, Tomsk, Russia: 264269. https://doi.org/10.1109/SIBCON.2009.5044868

Gazizov TR, Zabolotsky AM, Melkozerov AO, Dolganov ES, and Orlov PE (2012b). Improved design of modal filter for electronics protection. In the International Conference on lightning protection, IEEE, Vienna, Austria: 1-4. https://doi.org/10.1109/ICLP.2012.6344339

Gilb JP and Balanis CA (1989). Pulse distortion on multilayer coupled microstrip lines. IEEE Transactions on Microwave Theory and Techniques, 37(10): 1620-1628.

Kosowsky L (2012). Semiconductor devices including voltage switchable materials for over-voltage protection. U.S. Patent $7,923,844$

Krasonperkin VM, Samokhin GS, and Silin RA (1983a). Coupled lines. Electronic Technology, 6(354): 29-33.

Krasonperkin VM, Samokhin GS, and Silin RA (1983b). Pulse signals in coupled transmission lines. Electronic Technology, 7(355): 3-8.

Muthana P and Kroger H (2007). Behavior of Short Pulses on Tightly Coupled Microstrip Lines and Reduction of Crosstalk by Using Overlying Dielectric. IEEE Transactions on Advanced Packaging, 30(3): 511-520.

Orlov PE, Gazizov TR and Zabolotsky AM (2013). Experimental confirmation of the possibility for contactless diagnostics of multiconductor structures using modal probing. Russian Physics Journal, 56(6): 652-656.

Pavel V, Bulat K, Volkov N, and Tamara YI (2016). Interaction of a Shock Wave with a Cloud of Particles. IEJME-Mathematics Education, 11(8):2949-2962.

Senthinathan R, Prince JL, and Scheinfein MR (1987). Characteristics of coupled buried microstrip lines by modeling and simulation. IEEE Transactions on Components, Hybrid, and Manufacturing Technology, 10(4): 604-611.

Zabolotsky AM, Gazizov TR, Bova AG, and Radasky WA (2006). Dangerous pulse excitation of coupled lines. In the 17 th International Zurich Symposium on Electromagnetic Compatibility (EMC-Zurich '06), IEEE: 164-167. https://doi.org/10.1109/EMCZUR.2006.214895 